\title{
An Integral-type Constraint Qualification to Guarantee Nondegeneracy of the Maximum Principle for Optimal Control Problems with State Constraints ${ }^{\text {th }}$
}

\author{
S. O. Lopes ${ }^{\mathrm{a}}$, F. A. C. C. Fontes ${ }^{\mathrm{b}}$, M. d. R. de Pinho ${ }^{\mathrm{b}}$ \\ ${ }^{a}$ CMAT and Department of Mathematics and Applications, University of Minho \\ 4800-058 Guimarães, Portugal; \\ ${ }^{b}$ ISR-Porto, Faculdade de Engenharia, Universidade do Porto \\ 4200-465 Porto, Portugal
}

\begin{abstract}
For optimal control problems involving ordinary differential equations and functional inequality state constraints, the maximum principle may degenerate, producing no useful information about minimizers. This is known as the degeneracy phenomenon. Several non-degenerate forms of the maximum principle, valid under different constraint qualifications, have been proposed in the literature.

In this paper we propose a new constraint qualification under which a nondegenerate maximum principle is validated. In contrast with existing results, our constraint qualification is of an integral type. An advantage of the proposed constraint qualification is that it is verified on a larger class of problems with nonsmooth data and convex velocity sets.
\end{abstract}

Keywords: optimal control; maximum principle; state constraints; degeneracy; nonsmooth analysis. 2000 MSC: 49K15

\section{INTRODUCTION}

Since the birth of optimal control theory, commonly assumed to be in the late 50's of last century ([1]), the maximum principle has been a powerful and widely used analytic tool. As it is well known, maximum principles provide a set of necessary optimality conditions useful to identify, among admissible solutions, candidates to minimizers. The original statement of the maximum principle presented by Pontryagin et $a l$. has been generalized, strengthened and extended in many different ways. A major driving force behind these and other developments in optimal control theory has been the increasing number of applications.

Since state constraints are repeatedly encountered in applications, it is no surprise that the state constrained maximum principles have been the focus of intense research. Particularly relevant for our context is the work of Dubovitskii and Milyutin [2], which introduced measures in the maximum principle for such problems, and its extension to nonsmooth problems by Vinter and Pappas [3]. The state constrained maximum principle may exhibit a troublesome shortcoming. Indeed, and as it is amply illustrated by an example by Dubovitskii (see description in [4] and references therein), the maximum principle may degenerate if one end of the optimal trajectory belongs to the boundary of the state constraints. This phenomenon is known in the literature as the degeneracy phenomenon of the maximum principle for state constrained problems. It may arise in applications, most notably when Model Predictive Control frameworks are used (see e.g. [5] for a description of this technique) since the optimal control problems have to be solved for several initial states along the trajectory.

\footnotetext{
The financial support of FCT Project PTDC/EEA-CRO/116014/2009 "Optimal Control in Constrained and Hybrid Nonlinear Systems", European Union FP7 (FP7-PEOPLE-2010-ITN, Grant Agreement no. 264735-SADCO) and Project PEstC/MAT/UI0013/2011 is gratefully acknowledged

Email addresses: sofialopes@math.uminho.pt (S. O. Lopes), faf@fe.up.pt (F. A. C. C. Fontes), mrpinho@fe.up.pt (M. d. R. de Pinho) 
Clearly the occurrence of the degeneracy phenomenon has as a consequence that the necessary optimality conditions no longer give useful information to select minimizers. To remedy such a situation several authors have come up with conditions designed to identify classes of problems for which the maximum principle is nondegenerate (see, for example, [6], [7], [8], [9], [10], [11], [12], [13], etc). Other situations that may be related to the degeneracy phenomenon as normality and regularity of the optimal control and multipliers have also been amply studied; in this respect see [14], [15], [16], [17], [18], [19], [20], [21], [22], [23] among others.

In the literature, the conditions imposed to avoid the degeneracy phenomenon, called constraint qualifications, are inward pointing type conditions of mainly two types (see [13] for a discussion). One type of these conditions assumes knowledge of the optimal control. Although this type of conditions holds under less regularity assumptions, it has the disadvantage of being difficult to verify since the optimal control is not known a priori.

In this paper we focus on necessary conditions of optimality for state constrained problems. We propose a new and weaker type of inward pointing conditions to avoid the occurrence of the degeneracy phenomenon of the state constrained maximum principle. Differing from the literature, our constraint qualification is of integral-type (a preliminary version of these results was announced in $[24,25]$ ).

Our constraint qualification is a condition that implies, but is not implied by the constraint qualification in [9]. The accompanying nondegenerate maximum principle applies to problems with possibly nonsmooth data. The price we pay is that convexity of the so-called "velocity set" is assumed. Therefore, the results proposed here, can be applied to a larger class of problems with nonsmooth data and convex velocity sets.

This paper is organized as follows. We start by giving the main concepts and notation that are used throughout the paper in the next section. In Section 2 we describe, in the context of our results, optimal control problems with state constraints, the maximum principle, the degeneracy phenomenon and the literature on constraints qualifications designed to avoid the degeneracy phenomenon. Our integral type constraint qualification as well as the statement of the associated nondegenerate maximum principle is introduced and discussed in Section 3 where we state a smooth version of our main result. Section 4 focuses on the nonsmooth more general case. The proof of our main result is in Section 5.

\section{NECESSARY CONDITIONS OF OPTIMALITY AND THE DEGENERACY PHENOMENON}

Consider an optimal control problem with fixed initial state and with pathwise constraints:

$$
(P) \quad\left\{\begin{array}{lll}
\text { Minimize } & g(x(1)) & \\
\text { subject to } & & \text { a.e. } t \in[0,1] \\
& x(0)=x_{0}(t, x(t), u(t)) & \\
& x(1) \in C & \\
& u(t) \in \Omega(t) & \text { a.e. } t \in[0,1] \\
& h(t, x(t)) \leq 0 & \text { for all } t \in[0,1] .
\end{array}\right.
$$

The data for this problem comprises functions $g: \mathbb{R}^{n} \mapsto \mathbb{R}, f: \mathbb{R} \times \mathbb{R}^{n} \times \mathbb{R}^{m} \mapsto \mathbb{R}^{n}, h: \mathbb{R} \times \mathbb{R}^{n} \mapsto \mathbb{R}$, and a multifunction $\Omega:[0,1] \rightrightarrows \mathbb{R}^{m}$. The set of control functions for $(P)$ is $\mathcal{U}:=\left\{u:[0,1] \mapsto \mathbb{R}^{m}\right.$ : $u$ is a measurable function, $u(t) \in \Omega(t)$ a.e. $t \in[0,1]\}$. The state trajectory is an absolutely continuous function which satisfies the differential equation for some control function $u$. The domain of the above optimization problem is the set of admissible processes, namely pairs $(x, u)$ comprising a control function $u$ and a corresponding state trajectory $x$ which satisfy the constraints of $(P)$. We say that an admissible process $(\bar{x}, \bar{u})$ is a local minimizer if there exists $\delta>0$ such that $g(\bar{x}(1)) \leq g(x(1))$ for all admissible processes $(x, u)$ satisfying $\|x-\bar{x}\|_{L^{\infty}} \leq \delta$.

The MP for problems with state constraints, featuring measures as the multipliers associated with the such constraints, were first introduced by Dubovitskii and Milyutin in [2]. Several generalizations were developed, see for example $[26,27,28]$.

Assume that, for some $\delta^{\prime}>0$, the following hypotheses are satisfied. 
H1 The function $(t, u) \mapsto f(t, x, u)$ is $\mathcal{L} \times \mathcal{B}^{m}$ measurable for each $x$.

H2 There exists a $\mathcal{L} \times \mathcal{B}^{m}$ measurable function $k(t, u)$ such that $t \mapsto k(t, \bar{u}(t))$ is integrable and

$$
\left\|f(t, x, u)-f\left(t, x^{\prime}, u\right)\right\| \leq k(t, u)\left\|x-x^{\prime}\right\|
$$

for $x, x^{\prime} \in \bar{x}(t)+\delta^{\prime} \mathbb{B}, u \in \Omega(t)$ a.e. $t \in[0,1]$. There exist scalars $K_{f}>0$ and $\epsilon^{\prime}>0$ such that

$$
\left\|f(t, x, u)-f\left(t, x^{\prime}, u\right)\right\| \leq K_{f}\left\|x-x^{\prime}\right\|,
$$

for $x, x^{\prime} \in \bar{x}(0)+\delta^{\prime} \mathbb{B}, u \in \Omega(t)$ a.e. $t \in\left[0, \epsilon^{\prime}\right]$.

H3 The function $g$ is Lipschitz continuous on $\bar{x}(1)+\delta^{\prime} \mathbb{B}$.

H4 The graph of $\Omega$ is $\mathcal{L} \times \mathcal{B}^{m}$ measurable.

H5 The set $C$ is closed.

H6 The function $h$ is upper semicontinuous in $t$ and there exists a scalar $K_{h}>0$ such that

$$
\left|h(t, x)-h\left(t, x^{\prime}\right)\right| \leq K_{h}\left\|x-x^{\prime}\right\|,
$$

for all $t \in[0,1]$.

H7 There exist positive constants $\epsilon$ and $\epsilon_{1}$ such that $f(t, x, \Omega(t))$ is convex for all $t \in[0, \epsilon)$ and for all $x \in x_{0}+\epsilon_{1} \mathbb{B}$.

Here $\mathbb{B}$ denotes the closed unit ball and $\mathcal{L} \times \mathcal{B}^{m}$ denotes the product $\sigma$-algebra generated by Lebesgue sets of $[0,1]$ and Borel subsets of $\mathbb{R}^{m}$.

To simplify the exposition we start to present in this section and in section 3 the smooth case. Thus we add the following additional interim hypotheses:

AH2 The function $x \mapsto f(t, x, u)$ is continuously differentiable for each $(t, u)$.

AH3 The function $g$ is continuous differentiable on $\bar{x}(1)+\delta^{\prime} \mathbb{B}$.

AH5 The set $C$ is convex.

AH6 The function $x \mapsto h(t, x)$ is differentiable for fixed $t$ and $h$ and $h_{x}$ are continuous.

These hypotheses will be removed later in the main result, in section 4 .

The maximum principle (MP) for state constraints typically asserts existence of an absolutely continuous function $p$, a nonnegative regular Borel measure $\mu \in C^{*}([0,1], \mathbb{R})$, and a scalar $\lambda \geq 0$ satisfying

$$
\begin{gathered}
\mu\{[0,1]\}+\|p\|_{L^{\infty}}+\lambda>0, \\
-\dot{p}(t)=\left(p(t)+\int_{[0, t)} h_{x}(s, \bar{x}(s)) \mu(d s)\right) \cdot f_{x}(\bar{x}(t), \bar{u}(t)) \quad \text { a.e. } t \in[0,1], \\
-\left(p(1)+\int_{[0,1]} h_{x}(s, \bar{x}(s)) \mu(d s)\right) \in N_{C}(\bar{x}(1))+\lambda g_{x}(\bar{x}(1)), \\
\quad \operatorname{supp}\{\mu\} \subset\{t \in[0,1]: h(t, \bar{x}(t))=0\},
\end{gathered}
$$

and for almost every $t \in[0,1], \bar{u}(t)$ maximizes over $\Omega(t)$

$$
u \mapsto\left(p(t)+\int_{[0, t)} h_{x}(s, \bar{x}(s)) \mu(d s)\right) \cdot f(\bar{x}(t), u),
$$


where supp $\{\mu\}$ denotes the support of measure $\mu, C^{*}$ the dual space to the space of continuous functions and $N_{C}(x)$ denotes the normal cone to $C$ at $x$. (For convex sets the normal cone is simply $N_{C}(x):=\{y \in$ $\left.\mathbb{R}^{n}: y^{T}\left(x^{\prime}-x\right) \leq 0, x^{\prime} \in C\right\}$. For nonconvex sets it will be defined later in Section 4.)

This MP might not supply any useful information to select minimizers for certain optimal control problems where the trajectory starts on the boundary of the admissible state region, i.e. , when $h\left(0, x_{0}\right)=0$. This phenomenon arises if the multipliers take a certain form. If $h\left(0, x_{0}\right)=0$, the choice of degenerate multipliers ${ }^{1}$

$$
\lambda=0, \quad \mu \equiv \beta \delta_{\{t=0\}}, \quad \text { and } p \equiv-\beta h_{x}\left(0, x_{0}\right), \text { for some } \beta>0
$$

satisfy the necessary conditions but gives no information whatsoever about the optimal solution (note that the expression $p(t)+\int_{[0, t)} h_{x}(s, \bar{x}(s)) \mu(d s)$ vanishes and the conditions (1)-(5) are always satisfied). In this case, the necessary conditions of optimality $(\mathrm{NCO})$ are said to degenerate.

In order to avoid the degeneracy phenomenon, the NCO can be strengthened with additional conditions, typically a stronger form of the nontriviality condition (1). An example of such condition is

$$
\mu\{(0,1]\}+\|q\|_{L^{\infty}}+\lambda>0 .
$$

However, for such stronger form of the NCO to remain valid it is important to guarantee that we are not addressing problems for which the degenerate multipliers are the only possible choice. Such pathological problems were identified (see Dubovitskii example in [8]). Then, we need a constraint qualification asserting that the problem allows a set of multipliers in addition to the degenerate ones.

Different approaches to degenerate problems are reported in the literature. They mainly differ on the type of constraint qualification (CQ), as well as the remaining set of hypotheses under which the nondegenerate $\mathrm{NCO}$ are valid.

In the literature, the CQ to avoid degeneracy in optimal control problems are, typically, of two types:

CQ1: $\exists \delta, \epsilon>0$ and a control function $\hat{u}$ :

$$
h_{x}\left(s, x_{0}\right) \cdot\left[f\left(t, x_{0}, \hat{u}(t)\right)-f\left(t, x_{0}, \bar{u}(t)\right]<-\delta \quad \text { a.e. } s, t \in[0, \epsilon) .\right.
$$

Loosely speaking, this requires that there exist a control function pulling the state away from the boundary of the state constraint set faster than the optimal control on a neighborhood of the initial time.

CQ2: $\exists \delta, \epsilon>0$ and a control function $\hat{u}$ :

$$
h_{x}\left(s, x_{0}\right) \cdot f\left(t, x_{0}, \hat{u}(t)\right)<-\delta \quad \text { a.e. } s, t \in[0, \epsilon) .
$$

While CQ2 requires the existence of a control function pulling the state away from the state constraint boundary on a neighborhood of the initial time, CQ1 requires the existence of a control function pulling the state away from the state constraint boundary faster than the optimal control.

Extending CQ1 and CQ2 so that they hold on neighborhoods of each instant where the minimizing trajectory touches the boundary, leads to versions of MP in normal form, i.e. when the multiplier $\lambda$ equals to 1. Clearly, a normal form of MP implies a nondegenerate form of MP. However most of these results require extra regularity on data, see for example ([7], [10], [29], [30] and [31]).

The results using constraint qualification of the type CQ1 are not so easy to verify but are typically applicable to problems with less regularity on the data. In [13], we review several of such results and provide conditions under which CQ1 can be reduced to CQ2. Some recent results on this subject which are not discussed in [13] are in [20,32, 29].

The nondegenerate $\mathrm{NCO}$ we propose here are valid under a different set of constraint qualification; it is of integral-type and it holds for a larger class of problems.

\footnotetext{
${ }^{1}$ Here $\delta_{\{t=0\}}$ denotes Dirac unit measure concentrated at 0 .
} 


\section{AN INTEGRAL-TYPE CONSTRAINT QUALIFICATION}

In this section we provide our main result: it is a strengthened form of the MP valid under a new type of constraint qualification that we call integral-type CQ.

As it will be clear shortly our result is a modification of that in [9] that can be applied to a wider class of nonsmooth problems. To simplify the exposition from now on we will refer to the $C Q$ in [9] as $\mathbf{C Q}_{\mathbf{F F v 9 9}}$ and we denote by $\mathbf{C Q}_{\mathbf{I}}$ the integral-type $\mathrm{CQ}$ we propose here.

Consider the following constraint qualifications:

$\mathbf{C Q}_{\mathbf{I}}$ : if $h\left(0, x_{0}\right)=0$, then there exist positive constants $K_{u}, \epsilon, \epsilon_{1}, \delta$ and a control function $\hat{u} \in \mathcal{U}$ such that for all $t \in[0, \epsilon)$

$$
\int_{0}^{t} h_{x}(s, x(s)) \cdot\left[f\left(\tau, x_{0}, \hat{u}(\tau)\right)-f\left(\tau, x_{0}, \bar{u}(\tau)\right)\right] d \tau \leq-\delta t,
$$

for all $s \in[0, \epsilon), \quad x \in x_{0}+\epsilon_{1} \mathbb{B}$ and $\left\|f\left(t, x_{0}, \bar{u}(t)\right)\right\| \leq K_{u}$ and $\left\|f\left(t, x_{0}, \hat{u}(t)\right)\right\| \leq K_{u}$ a.e. $t \in[0, \epsilon)$.

CQFFV99: if $h\left(0, x_{0}\right)=0$, then there exist positive constants $K_{u}, \epsilon, \epsilon_{1}, \delta$ and a control function $\hat{u} \in \mathcal{U}$ such that for a.e. $t \in[0, \epsilon)$

$$
h_{x}(s, x(s)) \cdot\left[f\left(t, x_{0}, \hat{u}(t)\right)-f\left(t, x_{0}, \bar{u}(t)\right)\right]<-\delta,
$$

for all $s \in[0, \epsilon), x \in x_{0}+\epsilon_{1} \mathbb{B}$, and $\left\|f\left(t, x_{0}, \bar{u}(t)\right)\right\| \leq K_{u},\left\|f\left(t, x_{0}, \hat{u}(t)\right)\right\| \leq K_{u}$, a.e. $t \in[0, \epsilon)$.

Here and throughout, we denote by $\|\cdot\|$ the Euclidean norm.

Next we compare $\mathbf{C Q}_{\mathbf{I}}$ with $\mathbf{C} \mathbf{Q}_{\mathbf{F F V 9 9}}$. It is an easy task to see that $\mathbf{C Q}_{\mathbf{F F V 9 9}}$ implies $\mathbf{C Q}_{\mathbf{I}}$. However $\mathbf{C Q}_{\mathbf{I}}$ does not imply $\mathbf{C Q}_{\mathbf{F F V 9 9}}$. The following example describes a problem for which $\mathbf{C Q}_{\mathbf{I}}$ is verified, but CQFFv9 is not.

Example 3.1. Consider the problem

$$
\left(P_{E}\right)\left\{\begin{array}{cll}
\text { Minimize } & -x(1) & \\
\text { subject to } & \dot{x}(t)=u(t) & \text { a.e. } t \in[0,1] \\
& x(0)=0 & \\
& x(1) \in \mathbb{R} & \text { a.e. } t \in[0,1] \\
& u(t) \in \Omega(t) & \text { for all } t \in[0,1], \\
& x(t) \leq 0 &
\end{array}\right.
$$

where $\Omega(t)=\{u \in \mathbb{R}: l(t) \leq u \leq 0\}$ and $l$ is the function (see Fig. 1):

$$
l(t)=\left\{\begin{aligned}
&-1, t=0 \\
& 2-\frac{4}{2^{n}} t, t \in\left[\frac{1}{2} 2^{-n}, \frac{3}{4} 2^{-n}\right), n \in \mathbb{N} \\
& \frac{4}{2^{n}} t-4, \quad t \in\left[\frac{3}{4} 2^{-n}, 2^{-n}\right), n \in \mathbb{N}
\end{aligned}\right.
$$

The optimal solution to this problem is obtained by choosing $\bar{u}(t)=0$ and $\bar{x}(t)=0$ (u is always nonpositive). In order to test the two constraint qualifications, observe that

$$
h_{x}(s, x(s)) \cdot\left[f\left(t, x_{0}, \hat{u}(t)\right)-f\left(t, x_{0}, \bar{u}(t)\right)\right]=\hat{u}(t) .
$$

Thus, when applied to our problem, $\mathbf{C Q}_{\mathbf{F F V 9 9}}$ reduces to:

$$
\begin{aligned}
& \exists \delta, \epsilon>0 \text { and a control function } \hat{u}(t) \in \Omega(t) \text { such that for a.e. } t \in[0, \epsilon) \text { : } \\
& \qquad \hat{u}(t)<-\delta \quad \text { a.e. } t \in[0, \epsilon)
\end{aligned}
$$




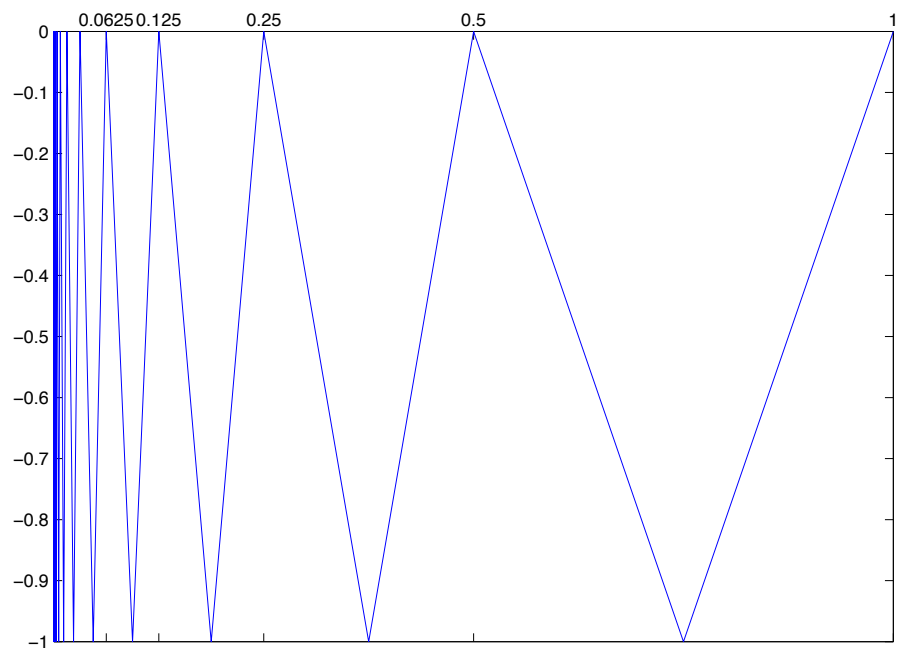

Figure 1: Function $t \mapsto l(t)$ defining for each $t$ the lower limit of the set $\Omega(t)$.

For any $\epsilon>0, l(t)=0$ and $\Omega(t)=\{0\}$ for a countable number of points $t \in[0, \epsilon)$. So CQFFv99 cannot be satisfied.

We now turn to $\mathbf{C Q}_{\mathbf{I}}$. This condition can also be reduced to:

$\exists \delta, \epsilon>0$ and a control function $\hat{u}(t) \in \Omega(t)$ such that for a.e. $t \in[0, \epsilon)$

$$
\int_{0}^{t} \hat{u}(\tau) d \tau \leq-\delta t \quad \forall t \in[0, \epsilon)
$$

We shall show that considering $\hat{u}(t)=l(t)$ then $\mathbf{C Q}_{\mathbf{I}}$ is satisfied.

For any $t \in(0,1]$ we can find an (unique) $k=2^{n}, n \in \mathbb{N}$ such that

$$
\frac{1}{2 k} \leq t \leq \frac{1}{k}
$$

Now

$$
\int_{0}^{t} l(s) d s=\int_{0}^{\frac{1}{2 k}} l(s) d s+\int_{\frac{1}{2 k}}^{t} l(s) d s .
$$

It can be seen that the first term is equal to $-\frac{1}{4 k}$ and the second term is negative. So, since

$$
\int_{0}^{t} l(s) d s \leq-\frac{1}{4 k} \leq-\frac{1}{4} t
$$

$\mathbf{C Q}_{\mathbf{I}}$ is satisfied.

Our constraint qualification $\mathbf{C Q}_{\mathbf{I}}$ is thus a weaker $C Q$ than $\mathbf{C Q}_{\mathbf{F F v 9 9}}$. Consequently, it applies to a larger class of problems. However, strengthening of necessary conditions under $\mathbf{C Q}_{\mathbf{I}}$ comes with a price: we need to assume that the velocity set is convex.

We now state a smooth version of our main result. Its nonsmooth counterpart, a more general result, is stated in the the next section. 
Theorem 3.2. Let $(\bar{x}, \bar{u})$ be a local minimizer. Assume that hypotheses $\mathbf{H 1}-\mathbf{H 6}$, the interim hypotheses AH2, AH3, AH5, AH6 and $\mathbf{C Q}_{\mathbf{I}}$ are satisfied. Then, the conditions (2)-(5) of the maximum principle hold, with the non-triviality condition replaced with

$$
\mu\{(0,1]\}+\|q\|_{L^{\infty}}+\lambda>0 .
$$

The above theorem is a corollary of our more general result stated below.

\section{THE NONSMOOTH RESULT}

Here, we make reference to $W^{1,1}$, the space of absolutely continuous functions, $C^{1,1}$, the space of continuously differentiable functions with locally Lipschitz continuous derivatives and co $(S)$ the convex hull of a set $S$.

Next we state some important concepts of nonsmooth analysis.

The limiting normal cone of a closed set $C \subset \mathbb{R}^{n}$ at $\bar{x} \in C$, denoted by $N_{C}(\bar{x})$, is the set

$$
\begin{aligned}
& N_{C}(\bar{x}):=\left\{\eta \in \mathbb{R}^{n}: \exists \text { sequences }\left\{M_{i}\right\} \in \mathbb{R}^{+}, x_{i} \rightarrow \bar{x}, \eta_{i} \rightarrow \eta\right. \text { such that } \\
& \left.x_{i} \in C \text { and } \eta_{i} \cdot\left(y-x_{i}\right) \leq M_{i}\left\|y-x_{i}\right\|^{2} \text { for all } y \in C, i=1,2, \ldots\right\} .
\end{aligned}
$$

The limiting subdifferential of a lower semicontinuous function $f: \mathbb{R}^{n} \rightarrow \mathbb{R} \cup\{\infty\}$ at a point $\bar{x} \in \mathbb{R}^{n}$ such that $f(\bar{x})<+\infty$, denoted by $\partial f(\bar{x})$, is defined to be

$$
\partial f(\bar{x})=\left\{\eta \in \mathbb{R}^{n}:(\eta,-1) \in N_{\text {epif }}(\bar{x}, f(\bar{x}))\right\} ;
$$

where epi $f=\left\{(x, \alpha) \in \mathbb{R}^{n+1}: \alpha \geq f(x)\right\}$ denotes the epigraph of a function $f$.

Here $\partial_{x}^{>} h(t, x)$, denotes the hybrid partial subdifferential of $h$ in the $x$-variable defined as

$$
\begin{gathered}
\partial_{x}^{>} h(t, x)=\operatorname{co}\left\{\xi: \quad \text { there exist }\left(t_{i}, x_{i}\right) \mapsto(t, x) \text { s.t. } h\left(t_{i}, x_{i}\right)>0,\right. \\
\left.h\left(t_{i}, x_{i}\right) \mapsto h(t, x), \text { and } h_{x}\left(t_{i}, x_{i}\right) \mapsto \xi\right\} .
\end{gathered}
$$

See [27] for a review of Nonsmooth Analysis and related concepts using a similar notation.

We now go back to the problem of interest. Let us consider the following constraint qualification:

$\mathbf{C Q}_{\mathbf{I}}^{\prime}$ : if $h\left(0, x_{0}\right)=0$, then there exist positive constants $K_{u}, \epsilon, \epsilon_{1}, \delta$ and a control function $\hat{u}(t) \in \Omega(t)$ such that for all $t \in[0, \epsilon)$

$$
\left\|f\left(t, x_{0}, \bar{u}(t)\right)\right\| \leq K_{u}, \quad\left\|f\left(t, x_{0}, \hat{u}(t)\right)\right\| \leq K_{u}
$$

and

$$
\int_{0}^{t} \zeta \cdot\left[f\left(\tau, x_{0}, \hat{u}(\tau)\right)-f\left(\tau, x_{0}, \bar{u}(\tau)\right)\right] d \tau \leq-\delta t
$$

for all $\zeta \in \partial_{x}^{>} h(s, x), s \in[0, \epsilon), x \in x_{0}+\epsilon_{1} \mathbb{B}$.

Observe that $\mathbf{C Q}_{\mathbf{I}}^{\prime}$ is a nonsmooth version of $\mathbf{C Q}_{\mathbf{I}}$.

Working upon a nonsmooth version of the MP presented in [27, Thm. 9.3.1] we get our main result that we now state.

Theorem 4.1. Let $(\bar{x}, \bar{u})$ be a local minimizer for $(P)$. Assume that, for some $\delta^{\prime}>0, \mathbf{C Q}_{\mathbf{I}}^{\prime}$ and the hypotheses $\mathbf{H 1}-\mathbf{H 6}$ are satisfied. Then there exist $p \in W^{1,1}\left([0,1]: \mathbb{R}^{n}\right)$, a measurable function $\gamma, a$ non-negative measure $\mu$ representing an element in $C^{*}([0,1]: \mathbb{R})$ and $\lambda \geq 0$ such that

$$
\begin{gathered}
\mu\{(0,1]\}+\|q\|_{L^{\infty}}+\lambda>0 \\
-\dot{p}(t) \in \operatorname{co} \partial_{x}(q(t) \cdot f(t, \bar{x}(t), \bar{u}(t))) \quad \text { a.e. } t \in[0,1],
\end{gathered}
$$




$$
\begin{gathered}
-q(1) \in N_{C}(\bar{x}(1))+\lambda \partial g(\bar{x}(1)), \\
\gamma(t) \in \partial_{x}^{>} h(t, \bar{x}(t)) \quad \mu \text { a.e. }, \\
\operatorname{supp}\{\mu\} \subset\{t \in[0,1]: h(t, \bar{x}(t))=0\},
\end{gathered}
$$

and, for almost every $t \in[0,1], \bar{u}(t)$ maximizes over $\Omega(t)$

$$
u \mapsto q(t) \cdot f(t, \bar{x}(t), u(t)),
$$

where

$$
q(t)= \begin{cases}p(t)+\int_{[0, t)} \gamma(s) \mu(d s) & t \in[0,1) \\ p(t)+\int_{[0,1]}^{\gamma} \gamma(s) \mu(d s) & t=1\end{cases}
$$

\section{PROOF OF THE MAIN RESULT}

The proof follows some of the steps of the approach in ([9]), i.e., we consider a sequence of approximating problems differing from $(P)$ in the dynamics near the left endpoint. However, while in [9] we consider variations of the controls, here we consider variations of the velocities.

We assume that $h\left(0, x_{0}\right)=0$, since otherwise the conditions of Theorem 4.1 cannot be satisfied with the trivial multipliers.

Consider, for $\alpha \in(0,1]$, absolutely continuous functions $x$ and $y$ satisfying the system of equations

$$
(S) \begin{cases}\dot{x}(t)=f(t, x(t), \bar{u}(t))+y(t) \cdot \Delta f(t, x(t)) & \text { a.e. } t \in[0, \alpha] \\ x(0)=x_{0}, & t \in[0, \alpha] \\ \dot{y}(t)=0 & \\ y(0) \in[0,1] & \end{cases}
$$

where $\Delta f(t, x):=f(t, x, \hat{u}(t))-f(t, x, \bar{u}(t))$.

Here $\hat{u}$ is the control function featuring in the constraint qualification $\mathbf{C Q}_{\mathbf{I}}^{\prime}$.

Since $\dot{y}=0$ and $y$ is absolutely continuous, then $y$ is constant. In what follows, we denote the value of that function by $y$ instead of $y(t)$.

This system plays an important role in what follows. Before proceeding with main steps of the proof we state some auxiliary lemmas. The proof of the lemmas can be found in the appendix.

Lemma 5.1. Let $x$ and $y$ be the solution of the system $(S)$ and $\bar{x}$ the minimizer of the $(P)$. There exist positive constants $A$ and $B$ such that, for $\alpha$ small enough, for all $t \in[0, \alpha]$

$$
\left\|x(t)-x_{0}\right\| \leq A t \text {, and }\|x(t)-\bar{x}(t)\| \leq B y t .
$$

Lemma 5.2. By reducing the size of $\alpha$, if necessary, we have that for any trajectory $x$ solving system (S)

$$
h(t, x(t)) \leq 0 \quad \text { for all } t \in[0, \alpha] .
$$

Now, take a decreasing sequence $\left\{\alpha_{i}\right\}$ on $(0, \alpha)$, converging to zero. Associate with each $\alpha_{i}$ the following problem $\left(P_{i}\right)$, where the state constraint is imposed only on the subinterval $\left[\alpha_{i}, 1\right]$ :

$$
\left(P_{i}\right)\left\{\begin{array}{lll}
\text { Minimize } & g(x(1)) & \\
\text { subject to } & \dot{x}(t)=f(t, x(t), \bar{u}(t))+y(t) \cdot \Delta f(t, x(t)) \\
& & \text { a.e. } t \in\left[0, \alpha_{i}\right) \\
& \dot{x}(t)=f(t, x(t), u(t)) & \text { a.e. } t \in\left[\alpha_{i}, 1\right] \\
& \dot{y}(t)=0 & t \in\left[0, \alpha_{i}\right) \\
& x(0)=x_{0} & \\
& x(1) \in C & \\
y(0) \in[0,1] & \text { a.e. } t \in\left[\alpha_{i}, 1\right] \\
u(t) \in \Omega(t) & \forall t \in[0,1] .
\end{array}\right.
$$


where

$$
\tilde{h}(t, x):= \begin{cases}r & t \in\left[0, \alpha_{i}\right) \\ h(t, x) & t \in\left[\alpha_{i}, 1\right]\end{cases}
$$

and $r$ is some fixed strict lower bound on the values of $h$.

We state the following Lemma.

Lemma 5.3. The trajectory $(x \equiv \bar{x}, y \equiv 0)$ is optimal for all problems $\left(P_{i}\right)$.

The Maximum Principle ([27, Thm. 9.3.1]) for the problem $\left(P_{i}\right)$ asserts the existence of an arc $\left(p_{i}, c_{i}\right)$ : $[0,1] \mapsto \mathbb{R}^{n} \times \mathbb{R}$, a measurable function $\gamma_{i}$, a nonnegative Radon measure $\mu_{i} \in C^{*}\left(\left[\alpha_{i}, 1\right], \mathbb{R}\right)$, and a scalar $\lambda_{i} \geq 0$ such that

$$
\begin{aligned}
& \mu_{i}\left\{\left[\alpha_{i}, 1\right]\right\}+\left\|\left(p_{i}, c_{i}\right)\right\|_{L^{\infty}}+\lambda_{i}>0, \\
& -\dot{p}_{i}(t) \in\left\{\begin{array}{l}
p_{i}(t) \cdot \operatorname{co} \partial_{x} f(t, \bar{x}(t), \bar{u}(t)), \text { a.e. } t \in\left[0, \alpha_{i}\right), \\
\left(p_{i}(t)+\int_{\left[\alpha_{i}, t\right)} \gamma_{i}(s) \mu_{i}(d s)\right) \cdot \operatorname{co} \partial_{x} f(t, \bar{x}(t), \bar{u}(t)), \text { a.e. } t \in\left[\alpha_{i}, 1\right],
\end{array}\right. \\
& -\dot{c}_{i}(t)= \begin{cases}p_{i}(t) \cdot \Delta f(t, \bar{x}(t)), & \text { a.e. } t \in\left[0, \alpha_{i}\right), \\
0, & \text { a.e. } t \in\left[\alpha_{i}, 1\right],\end{cases}
\end{aligned}
$$

for almost every $t \in\left[\alpha_{i}, 1\right], \bar{u}(t)$ maximizes over $\Omega(t)$

$$
\begin{gathered}
u \mapsto\left(p_{i}(t)+\int_{\left[\alpha_{i}, t\right)} \gamma_{i}(s) \mu_{i}(d s)\right) \cdot f(t, \bar{x}(t), u), \\
\operatorname{supp}\left\{\mu_{i}\right\} \subset\left\{t \in\left[\alpha_{i}, 1\right]: h(t, \bar{x}(t))=0\right\}, \\
\gamma_{i}(t) \in \partial_{x}^{>} h(t, \bar{x}(t)) \quad \mu \text { a.e. }
\end{gathered}
$$

for some $\xi_{i} \in \partial_{x} g(\bar{x}(1))$,

$$
\begin{gathered}
-\left(p_{i}(1)+\int_{\left[\alpha_{i}, 1\right]} \gamma_{i}(s) \mu_{i}(d s)\right) \in N_{C}(\bar{x}(1))+\lambda_{i} \xi_{i}, \\
-c_{i}(1)=0, c_{i}(0) \leq 0 .
\end{gathered}
$$

It remains to pass to the limit as $i \rightarrow \infty$ and thereby, obtain a set of nondegenerate multipliers for the original problem.

Without changing the notation, we extend $\mu_{i}$ as a regular Borel measure on $[0,1]$

$$
\mu_{i}(\mathcal{B})=\mu_{i}\left(\mathcal{B} \cap\left[\alpha_{i}, 1\right]\right) \text { for all Borel set } \mathcal{B} \subset[0,1] .
$$

Extend also $\gamma_{i}$, originally defined on $\left[\alpha_{i}, 1\right]$, arbitrarily to the interval $[0,1]$ as a Borel measurable function. With these extensions and noting that $\mu\left(\left[0, \alpha_{i}\right)\right)=0$ we can write

$$
-\dot{p}_{i}(t) \in\left(p_{i}(t)+\int_{[0, t)} \gamma_{i}(s) \mu_{i}(d s)\right) \cdot \operatorname{co} \partial_{x} f(t, \bar{x}(t), \bar{u}(t)) \text { a.e. } t \in[0,1] .
$$

It is easy to see that $c_{i}$ can be omitted from (16), since $p_{i} \equiv 0$ implies $c_{i} \equiv 0$. By scaling the multipliers we ensure that

$$
\left\|\mu_{i}\left\{\left[\alpha_{i}, 1\right]\right\}\right\|+\left\|p_{i}\right\|_{L^{\infty}}+\lambda_{i}=1 .
$$


By (19) and (20) we deduce that $\mu_{i} \rightarrow \mu$ weakly* for some $\mu$ in the space of nonnegative valued function in $C^{*}([0,1], \mathbb{R})$. The multifunction $\partial_{x}^{>} h$ is uniformly bounded, compact, convex, and has a closed graph. So, $\gamma_{i}(t)$ are uniformly bounded. It follows from Proposition 9.2.1 in [27] that, along a subsequence, $\gamma_{i} d \mu_{i} \rightarrow \gamma d \mu$ weakly *. By $(20),\left\{p_{i}\right\}$ is uniformly bounded. $\partial_{x} f(t, \bar{x}(t), u(t))$ is integrally bounded. It follows from the above that $\left\{\dot{p}_{i}\right\}$ are uniformly integrally bounded. By compactness results (see for example Theorem 2.5.3 of [27]), we deduce that along a subsequence $p_{i} \rightarrow p$ uniformly. It is a simple matter to see that $\lambda_{i} \rightarrow \lambda$ and by $H 3, \xi_{i} \rightarrow \xi$ along a subsequence.

It follows that the conditions $(2),(13),(4)$ for problem $(P)$ are satisfied and since $N_{C}(\bar{x}(1))$ is closed, (3) also holds. Moreover from (20) we deduce

$$
\mu\{[0,1]\}+\|p\|_{L^{\infty}}+\lambda=1 .
$$

Consider the set $S_{i}=\left[\alpha_{i}, 1\right] \backslash \mathcal{T}_{i}$ where $\mathcal{T}_{i}$ is a null Lebesgue measure set in $\left[\alpha_{i}, 1\right]$ containing all times where the maximization of (18) is not achieved at $\bar{u}$. We can then write

$$
\left(p_{i}(t)+\int_{\left[\alpha_{i}, t\right)} \gamma_{i}(s) \mu_{i}(d s)\right) \cdot f(t, \bar{x}(t), u) \leq\left(p_{i}(t)+\int_{\left[\alpha_{i}, t\right)} \gamma_{i}(s) \mu_{i}(d s)\right) \cdot f(t, \bar{x}(t), \bar{u}(t)),
$$

for all $t \in S_{i}$ and for all $u \in \Omega(t)$.

Now consider the full measure set $S=(0,1] \backslash \bigcup_{i} \mathcal{T}_{i}$. Fix some $t$ in $S$. Then for all $i>N$, where N is such that $\alpha_{N} \leq t$, we have

$$
\left(p_{i}(t)+\int_{[0, t)} \gamma_{i}(s) \mu_{i}(d s)\right) \cdot f(t, \bar{x}(t), u) \leq\left(p_{i}(t)+\int_{[0, t)} \gamma_{i}(s) \mu_{i}(d s)\right) \cdot f(t, \bar{x}(t), \bar{u}(t)) .
$$

for all $u \in \Omega(t)$. Applying limits to both sides of this inequality we obtain (15).

We have established that the set of multipliers $(p, \mu, \lambda)$, obtained as limit of $\left(p_{i}, \mu_{i}, \lambda_{i}\right)$ satisfy the conditions (2)-(5) for the original problem $(P)$ together with $(21)$.

It remains to verify

$$
\mu\{(0,1]\}+\|q\|_{L^{\infty}}+\lambda>0 .
$$

To do this, the following lemma will be useful.

Lemma 5.4. The adjoint vector $p_{i}$ in the necessary conditions of optimality for problem $\left(P_{i}\right)$ satisfies

$$
\int_{0}^{\alpha_{i}} p_{i}(t) \cdot \Delta f(t, \bar{x}(t)) d t \leq 0
$$

In view of the constraint qualification, there exists positive constants $\epsilon$ and $\delta$ such that for all $t \in[0, \epsilon)$

$$
\int_{0}^{t} \zeta \cdot\left[f\left(\tau, x_{0}, \hat{u}(\tau)\right)-f\left(\tau, x_{0}, \bar{u}(\tau)\right)\right] d \tau \leq-\delta t
$$

for all $\zeta \in \partial_{x}^{>} h(s, x), s \in[0, \epsilon), x \in x_{0}+\epsilon_{1} \mathbb{B}$.

Suppose to the contrary that

$$
\mu\{(0,1]\}+\|q\|_{L^{\infty}}+\lambda=0 .
$$

Due to (21), we must have

$$
\lambda=0, \quad \mu=\beta \delta_{\{0\}}, \quad p(t)=-\beta \zeta \quad \text { for some } \beta>0 \text { and } \zeta \in \partial_{x}^{>} h\left(0, x_{0}\right) .
$$

The constraint qualification $\left(\mathbf{C Q}_{\mathbf{I}}^{\prime}\right)$ implies

$$
\int_{0}^{t}-p(\sigma) \cdot \Delta f\left(\sigma, x_{0}\right) d \sigma=\int_{0}^{t} \beta \zeta \cdot \Delta f\left(\sigma, x_{0}\right) d \sigma \leq-\delta \beta t .
$$


On the other hand, using also Lemma 5.1, we have

$$
\begin{aligned}
\int_{0}^{\alpha_{i}} p_{i}(t) \cdot \Delta f(t, \bar{x}(t)) d t & =\int_{0}^{\alpha_{i}}\left(p(t) \cdot \Delta f\left(t, x_{0}\right)+\left(p_{i}(t)-p(t)\right) \cdot \Delta f\left(t, x_{0}\right)+p_{i}(t) \cdot\left[\Delta f(t, \bar{x}(t))-\Delta f\left(t, x_{0}\right)\right]\right) d t \\
& \geq \delta \beta \alpha_{i}-\int_{0}^{\alpha_{i}}\left(2 K_{u}\left\|p_{i}(t)-p(t)\right\|+2 K_{f} A t\left\|p_{i}(t)\right\|\right) d t .
\end{aligned}
$$

By the uniform convergence of $p_{i}$, we can make $\left\|p_{i}-p\right\|<\bar{\epsilon}$ for any $\bar{\epsilon}>0$ of our choice provided we choose a sufficient large $i$. Moreover $\left\|p_{i}\right\| \leq 1$.

It follows that

$$
\int_{0}^{\alpha_{i}} p_{i}(t) \cdot \Delta f(t, \bar{x}(t)) d t \geq \delta \beta \alpha_{i}-\left(2 K_{u} \bar{\epsilon} \alpha_{i}+K_{f} A \alpha_{i}^{2}\right)>\delta \beta / 2 \alpha_{i}>0
$$

if $\bar{\epsilon}<\frac{\delta \beta}{8 K_{u}}$ and $\alpha_{i}<\frac{\delta \beta}{4 K_{f} A}$.

So, we would have $\int_{0}^{\alpha_{i}} p_{i}(t) \cdot \Delta f(t, \bar{x}(t)) d t>0$ contradicting Lemma 5.4. We deduce (9).

\section{References}

[1] L. S. Pontryagin, V. G. Boltyanskii, R. V. Gamkrelidze, E. F. Mishchenko, The Mathematical Theory of Optimal Processes, Wiley Interscience, New York, 1962.

[2] A. Y. Dubovitskii, A. Milyutin, Extremum problems under constraints, Dokl. Akad. Nauk SSSR 149 (1963) $759-762$.

[3] R. B. Vinter, G. Pappas, A maximum principle for nonsmooth optimal-control problems with state constraints, J. Math. Anal. Appl. 89 (1) (1982) 212-232. doi:10.1016/0022-247X(82)90099-3. URL http://dx.doi.org/10.1016/0022-247X(82)90099-3

[4] A. V. Arutyunov, S. M. Aseev, State constraints in optimal control. The degeneracy phenomenon, Systems Control Lett. 26 (4) (1995) 267-273. doi:10.1016/0167-6911(95)00021-Z. URL http://dx.doi.org/10.1016/0167-6911(95)00021-Z

[5] F. A. C. C. Fontes, A general framework to design stabilizing nonlinear model predictive controllers, Systems Control Lett. 42 (2) (2001) 127-143. doi:10.1016/S0167-6911(00)00084-0. URL http://dx.doi.org/10.1016/S0167-6911(00) 00084-0

[6] A. V. Arutyunov, N. Tynyanskii, The maximum principle in a problem with phase constraints, Soviet J. Comput. Systems Sci. 23 (1985) 28-35.

[7] M. M. A. Ferreira, R. B. Vinter, When is the maximum principle for state constrained problems nondegenerate?, J. Math. Anal. Appl. 187 (2) (1994) 438-467. doi:10.1006/jmaa.1994.1366.

URL http://dx.doi.org/10.1006/jmaa.1994.1366

[8] A. V. Arutyunov, S. M. Aseev, Investigation of the degeneracy phenomenon of the maximum principle for optimal control problems with state constraints, SIAM Journal of Control and Optimization 35 (1997) 930-952.

[9] M. M. A. Ferreira, F. A. C. C. Fontes, R. B. Vinter, Nondegenerate necessary conditions for nonconvex optimal control problems with state constraints, J. Math. Anal. Appl. 233 (1) (1999) 116-129. doi:10.1006/jmaa.1999.6270. URL http://dx.doi.org/10.1006/jmaa.1999.6270

[10] F. Rampazzo, R. B. Vinter, A theorem on existence of neighbouring trajectories satisfying a state constraint, with applications to optimal control, IMA J. Math. Control Inform. 16 (4) (1999) 335-351.

[11] F. Rampazzo, R. Vinter, Degenerate optimal control problems with state constraints, SIAM J. Control Optim. 39 (4) (2000) 989-1007 (electronic). doi:10.1137/S0363012998340223. URL http://dx.doi.org/10.1137/S0363012998340223

[12] F. A. C. C. Fontes, Nondegenerate necessary conditions of optimality for control problems with state constraints, in: Nonlinear Control Systems (A. B. Kurzhanski and A. L. Fradkov, eds.), 2002, pp. 45-50.

[13] S. O. Lopes, M. de Pinho, F. A. C. C. Fontes, On constraint qualifications for nondegenerate necessary conditions of optimality applied to optimal control problems, Discrete and Continuous Dynamical Systems Series A (DCDS-A) 29 (2) (2011) $559-575$.

URL http://dx.doi.org/10.3934/dcds.2011.29.559

[14] J. F. Rosenblueth, Conditions for generic normality in optimal control, Systems and Control Letters 9 (5) (1987) 433 438. doi:10.1016/0167-6911(87)90073-9.

URL http://www.sciencedirect.com/science/article/pii/0167691187900739

[15] M. M. A. Ferreira, On the regularity of optimal controls for a class of problems with state constraints, International Journal of Systems Science 37 (8) (2006) 495-502.

[16] H. Frankowska, Regularity of minimizers and of adjoint states for optimal control problems under state constraints, J. Convex Analysis 13 (2006) 299-328. 
[17] H. Frankowska, Normality of the maximum principle for absolutely continuous solutions to bolza problems under state constraints, Control and Cybernetics 38 (2009) 1327-1340.

[18] H. Frankowska, E. M. Marchini, Lipschitzianity of optimal trajectories for the bolza optimal control problem, Calculus of Variations and Partial Differential Equations 27 (4) (2006) 467-492.

[19] G. N. Galbraith, R. B. Vinter, Lipschitz continuity of optimal controls for state constrained problems, SIAM Journal of Control and Optimization 42 (5) (2003) 1727-1774.

[20] H. Frankowska, M. Mazzola, Optimal synthesis and normality of the maximum principle for optimal control problems with pure state constraints, in: Proceedings of the 9th IEEE International Conference on Control and Automation (ICCA 2011), Santiago, Chile, 2011, pp. 945-950.

[21] R. B. Vinter, H. Zheng, Necessary conditions for optimal control problems with state constraints, Transactions of the American Mathematical Society 350 (3) (1998) 1181-1204.

[22] P. Bettiol, H. Frankowska, Lipschitz regularity of solution map of control systems with multiple state constraints, Discrete Contin. Dynam. Systems - A 32 (2012) 1-26.

[23] C. Gavriel, S. L. Lopes, R. B. Vinter, Regularity of minimizers for higher order variational problems in one independent variable, Annual Reviews in Control 35 (2011) 172-177.

[24] S. O. Lopes, F. A. C. C. Fontes, Nondegenerate necessary conditions for optimal control problems with state constraints: Integral-type constraint qualification, in: ECC'07 conference, Kos, Greece, 2007.

[25] S. O. Lopes, Nondegenerate forms of the maximum principle for optimal control problems with state constraints, Phd thesis, University of Minho, Guimaraes, Portugal, http://repositorium.sdum.uminho.pt/ (2009). URL http://hdl.handle.net/1822/8983

[26] F. H. Clarke, Optimization and nonsmooth analysis, Canadian Mathematical Society Series of Monographs and Advanced Texts, John Wiley \& Sons Inc., New York, 1983, a Wiley-Interscience Publication.

[27] R. Vinter, Optimal control, Birkhauser, Boston, 2000.

[28] M. d. R. de Pinho, M. M. A. Ferreira, F. A. C. C. Fontes, Unmaximized inclusion necessary conditions for nonconvex constrained optimal control problems, ESAIM:Control, Optimisation and Calculus of Variations 11 (4) (2005) 614-632.

[29] F. A. C. C. Fontes, S. O. Lopes, Normal forms of necessary conditions for dynamic optimization problems with pathwise inequality constraints, Journal of Mathematical Analysis and Applications 399 (1) 27-37.

[30] A. Cernea, H. Frankowska, A connection between the maximum principle and dynamic programming for constrained control problems, SIAM J. Control Optim. 44 (2) (2005) 673-703 (electronic). doi:10.1137/S0363012903430585. URL http://dx.doi.org/10.1137/S0363012903430585

[31] P. Bettiol, H. Frankowska, Normality of the maximum principle for nonconvex constrained Bolza problems, J. Differential Equations 243 (2) (2007) 256-269. doi:10.1016/j.jde.2007.05.005. URL http://dx.doi.org/10.1016/j.jde.2007.05.005

[32] A. V. Arutyunov, D. Karamsin, F. L. Pereira, The maximum principle for optimal control problems with state constraints by r.v. gramkrelidze: Revisited, Journal of Optimization Theory and Applications 149 (2011) 474-493.

[33] J. Warga, Optimal control of differential and functional equations, Academic Press, New York, 1972.

\section{Appendix A. Proof of Lemmas}

Proof. (of Lemma 5.1)

Take any $\alpha \in[0, \epsilon)$, where $\epsilon$ is defined in $\mathbf{C Q}_{\mathbf{I}}^{\prime}$. Integrating $x$ we have that

$$
\begin{aligned}
\left\|x(t)-x_{0}\right\| & \leq \int_{0}^{t}\|f(\tau, x(\tau), \bar{u}(\tau))+y \cdot \Delta f(\tau, x(\tau))\| d \tau \\
& \leq \int_{0}^{t} 3 K_{f}\left\|x(\tau)-x_{0}\right\| d \tau+3 K_{u} t .
\end{aligned}
$$

Applying Gronwall-Bellman inequality (see e.g. [33]) yields

$$
\begin{aligned}
\left\|x(t)-x_{0}\right\| & \leq 3 K_{u} t+e^{3 K_{f} t} \int_{0}^{t} 9 K_{f} K_{u} \tau d \tau \\
& =3 K_{u} t+\frac{9}{2} K_{f} K_{u} e^{3 K_{f} t} t^{2} .
\end{aligned}
$$

Since $0 \leq t \leq \alpha \leq 1$, we deduce that:

$$
\left\|x(t)-x_{0}\right\| \leq 3 K_{u} t+\frac{9}{2} K_{f} K_{u} e^{3 K_{f}} t=A t,
$$

where $A:=3 K_{u}+\frac{9}{2} K_{f} K_{u} e^{3 K_{f}}$. The first assertion of the lemma is proved. 
Similarly

$$
\begin{aligned}
\|x(t)-\bar{x}(t)\| & \leq \int_{0}^{t}\|f(\tau, x(\tau), \bar{u}(\tau))+y \cdot \Delta f(\tau, x(\tau))-f(\tau, \bar{x}(\tau), \bar{u}(\tau))\| d \tau \\
& \leq \int_{0}^{t}\left[K_{f}\|x(\tau)-\bar{x}(\tau)\|+2 y K_{f}\left\|x(\tau)-x_{0}\right\|\right] d \tau+2 y K_{u} t \\
& \leq \int_{0}^{t} K_{f}\|x(\tau)-\bar{x}(\tau)\| d \tau+y K_{f} A t^{2}+2 y K_{u} t .
\end{aligned}
$$

Applying Gronwell's Lemma

$$
\begin{aligned}
\|x(t)-\bar{x}(t)\| & \leq y K_{f} A t^{2}+2 y K_{u} t+e^{K_{f} t} \int_{0}^{t} K_{f} y\left[K_{f} A s^{2}+2 K_{u} s\right] d s \\
& =y K_{f} A t^{2}+2 y K_{u} t+y K_{f} e^{K_{f} t}\left(\frac{K_{f} A t^{3}}{3}+K_{u} t^{2}\right) .
\end{aligned}
$$

As $0 \leq t \leq 1$

$$
\|x(t)-\bar{x}(t)\| \leq B y t
$$

where $B:=K_{f} A+2 K_{u}+K_{f} e^{K_{f}}\left(K_{f} \frac{A}{3}+K_{u}\right)$, proving the second assertion.

Proof. (of Lemma 5.2)

Choose an $\alpha$ satisfying

$$
\alpha<\min \left\{\frac{2 \delta}{K_{h} K_{f}(2 A+B)}, \frac{\epsilon_{1}}{A}, \epsilon\right\} .
$$

Take any $x$ solving $(S)$ and suppose that for some fixed $t \in[0, \alpha]$

$$
h(t, x(t))>0 .
$$

Define, for $\beta \in[0,1]$,

$$
r(\beta):=h(t, \bar{x}(t)+\beta(x(t)-\bar{x}(t))) .
$$

In view of the properties of $h$ as a function of $x, r$ is continuous. We also have

$$
\begin{aligned}
& r(0)=h(t, \bar{x}(t)) \leq 0, \\
& r(1)=h(t, x(t))>0 .
\end{aligned}
$$

It follows that the set

$$
D:=\{\beta \in[0,1]: r(\beta)=0\}
$$

is non-empty, closed and bounded. We can therefore define

$$
\beta_{m}:=\max _{\beta \in D} \beta .
$$

Since $r(1)>0$, we have $\beta_{m}<1$. Take any $\beta \in\left(\beta_{m}, 1\right]$. Applying the Lebourg Mean-Value Theorem $([26])$, we obtain

$$
\begin{aligned}
h(t, x(t))-r(\beta) & =\zeta_{t} \cdot[x(t)-\bar{x}(t)-\beta(x(t)-\bar{x}(t))] \\
& =(1-\beta) \zeta_{t} \cdot[x(t)-\bar{x}(t)]
\end{aligned}
$$

for some $\zeta_{t} \in \operatorname{co} \partial_{x} h(t, \hat{x})$, and $\hat{x}$ in the segment $(x(t), \bar{x}(t)+\beta[x(t)-\bar{x}(t)])$. 
Since $r(\beta)>0$ for all $\beta \in\left(\beta_{m}, 1\right]$, we have that $h(t, \hat{x})>0$. Thus, in this case, $\operatorname{co} \partial_{x} h(t, \hat{x}) \subset \partial_{x}^{>} h(t, \hat{x})$, by the respective definitions. It follows that $\zeta_{t} \in \partial_{x}^{>} h(t, \hat{x})$.

Expanding the expression (A.3) yields

$$
\begin{aligned}
h(t, x(t))-r(\beta)= & (1-\beta) \zeta_{t} \cdot \int_{0}^{t}[f(\tau, x(\tau), \bar{u}(\tau))+y \Delta f(\tau, x(\tau))-f(\tau, \bar{x}(\tau), \bar{u}(\tau))] d \tau \\
\leq & (1-\beta)\left(\zeta _ { t } \cdot \int _ { 0 } ^ { t } y \left(\Delta f\left(\tau, x_{0}\right)+\Delta f(\tau, x(\tau))-\Delta f\left(\tau, x_{0}\right) d \tau\right.\right. \\
& \left.+\left\|\zeta_{t}\right\| K_{f} \int_{0}^{t}\|x(\tau)-\bar{x}(\tau)\| d \tau\right) \\
\leq & (1-\beta)\left(\int_{0}^{t} \zeta_{t} \cdot y \Delta f\left(\tau, x_{0}\right) d \tau+2 K_{f} K_{h} y \int_{0}^{t}\left\|x(\tau)-x_{0}\right\| d \tau\right. \\
& \left.+K_{h} K_{f} \int_{0}^{t}\|x(\tau)-\bar{x}(\tau)\| d \tau\right) \\
\leq & (1-\beta) y t\left(-\delta+K_{h} K_{f}(A+B / 2) t\right) \\
\leq & 0,
\end{aligned}
$$

for all $\beta \in\left(\beta_{m}, 1\right]$.

Here we have used the fact that the norm of every element of the subdifferential is bounded by the Lipschitz rank of the function.

In the last two inequalities we have used $\mathbf{C Q}_{\mathbf{I}}^{\prime}$ and (A.1).

Since $r$ is continuous and $r\left(\beta_{m}\right)=0$, it follows that

$$
h(t, x(t)) \leq 0
$$

This contradicts (A.2). The proof is complete.

Proof. (of Lemma 5.3)

By contradiction assume that there exist $(\hat{y}, \hat{x}) \neq(0, \bar{x})$ that solve $\left(P_{i}\right)$. Hence $g(\hat{x}(1))<g(\bar{x}(1))$ and $\dot{\hat{x}}(t)=f(t, \hat{x}(t), \bar{u}(t))+\hat{y} \cdot \Delta f(t, \hat{x}(t))$ a.e. $t \in\left[0, \alpha_{i}\right)$.

By convexity hypotheses (H7)

$$
\hat{y} f(t, \hat{x}, \hat{u})+(1-\hat{y}) f(t, \hat{x}, \bar{u}) \in f(t, x(t), \Omega(t)) .
$$

Then $\exists \hat{u}(\cdot):[0,1] \rightarrow \mathbb{R}^{m}:$

$$
\dot{\hat{x}}(t)=f(t, \hat{x}, \hat{u}(t)) \text { a.e. } t \in[0,1] .
$$

We conclude that $\hat{x}$ is an admissible trajectory for $(P)$ with $g(\hat{x}(1))<g(\bar{x}(1))$. 